\title{
Two hypotheses of Brugada Syndrome - Repolarization and Depolarization: Literature Review
}

\author{
Clair E. Koo ${ }^{1}$ and Ethan Hutt ${ }^{2}$ \\ ${ }^{1}$ Henry M. Gunn High School, Palo Alto, CA, USA \\ 'University of North Carolina at Chapel Hill, Chapel Hill, NC, USA \\ DOI: https://doi.org/10.47611/jsrhs.v10i2.1444
}

\section{ABSTRACT}

Brugada syndrome is a recently discovered cardiac disease. The mechanisms behind this syndrome are unknown, but scientists are able to hypothesize possible mechanisms through various studies of electrocardiograms, canine wedge preparations, and genetic mutations. This review goes over two of the most potential, highly debated hypotheses that explain the causes of the Brugada syndrome: the repolarization and depolarization hypotheses. Studies by Zhang et al., Wilde et al., Postema et al., Meregalli et al., Tse et al., and other scientists are reviewed to understand and evaluate the mechanisms behind each of the hypotheses. The mechanisms of both hypotheses are explained in detail. Published evidence helps us suggest that the most likely cause of the Brugada syndrome is the mechanism behind the repolarization hypothesis.

\section{Introduction}

In a normally functioning heart, electrical impulses travel along the heart through sodium $\left(\mathrm{Na}^{+}\right)$and potassium $\left(\mathrm{K}^{+}\right)$ channels in a process called action potential (AP). Brugada syndrome is a heart rhythm disorder that involves a mutation of the genes for the $\mathrm{Na}^{+}$and $\mathrm{K}^{+}$channels and other cardiac proteins. These mutations result in an abnormal heartbeat, mainly ventricular fibrillation. Ventricular fibrillation (VF) occurs when the heart muscle twitches randomly instead of completing the full action of pumping blood. This can be very dangerous because the heart is unable to provide sufficient blood to the brain.

The disorder is caused primarily by mutations to the SCN5A gene - a gene associated with cardiac $\mathrm{Na}^{+}$ channels located on chromosome 3 (NORD, 2016). A total of 19 genes have been linked to Brugada syndrome (Le Scouarnec et al., 2015). Each varying mutation also determines the severity of the syndrome and the gene's appearance as a phenotype (Tse et al., 2016). The mutations of genes also suggest that the syndrome is passed down from generation to generation as an autosomal dominant gene, with children having 50\% chance of inheriting the disorder; however, only $15 \%-30 \%$ of patients have a SCN5A mutation and $65 \%-70 \%$ of clinically diagnosed cases have no identified genetic cause (NORD, 2016). Only several mutations are identified in patients with Brugada syndrome, with many other mutations not being confirmed to be the root cause of the disorder. All mutations are not associated with Brugada syndrome and cannot determine whether the patient shows symptoms of the disorder. It increases the difficulty of diagnoses.

Symptoms of Brugada syndrome include dizziness, fainting/black outs (syncope), gasping (particularly at rest), irregular heartbeats (palpitations), extremely fast or chaotic heartbeat, seizures, and sudden cardiac arrest (NORD, 2016). In fact, the first symptom of many Brugada patients is cardiac arrest, making it very difficult to diagnose patients before the onset of symptoms. Blackouts are common during brief moments of abnormal rhythm.

Brugada syndrome can also occur along with other disorders such as the Long QT syndrome type 3 (LQT3) a form of Romano-Ward Syndrome, mainly due to overlapping similarities in mutations to the SCN5A gene (NORD, 2016). The Romano-Ward Syndrome is also an inherited cardiac disorder associated with electrical abnormalities of 
the heart (NORD, 2016). Families have reported that members have both Brugada syndrome and LQT3, possibly showing that Brugada syndrome and LQT3 are different types of the same disorder (Tse et al., 2016).

1 in 2,000 people are affected by the Brugada syndrome (NORD, 2016). Patients are born with the syndrome, but it is only once they have fully developed, or reached adulthood, when symptoms appear. It is rare to have symptoms occur in children, but when apparent, it is equally likely to be in both female and male (Mayo Clinic I, 2020). However, in adult patients, the disorder is more likely to occur in males (Tse et al., 2016). Cardiologists, who have patients with Brugada syndrome, test close family members like children once they reach age. In addition, the syndrome is more likely in people of Asian descent and is likely to be the most common reason for sudden unexpected nocturnal death syndrome (SUNDS) in many young Asian males (Tse et al., 2016). In fact, recent reports suggest that Brugada syndrome could be responsible for up to 2 out of 10 patients with sudden death and structurally normal hearts (NORD, 2016). This further supports that sudden death can be the first sign of the disease. In addition, although it doesn't cause people to get Brugada syndrome, a fever can increase heart rate and potentially cause the heart to enter VF and sudden cardiac arrest with someone with Brugada syndrome and even with a structurally normal heart; it also potentially affects children (Mayo Clinic II, 2020).

Josep and Pedro Brugada discovered the disorder in 1992, although in 1989 there was an association of sudden cardiac with the Brugada syndrome pattern on Electrocardiogram (ECG) (NORD, 2016). In 1998, Ramon Brugada identified the patterns resulting from the phenotype differences in Brugada patients as shown in Figure 1 (Larkin, 2020).

As the discovery of the disorder is recent and not much evidence has been collected in comparison to other syndromes, further tests are needed to pinpoint the reason for the Brugada syndrome. ECG patterns, genetic tests, animal models, and structural abnormalities are used to determine the mechanism behind the disease. Tests involving induction of the Brugada pattern through medication in potential Brugada syndrome patients have also been observed. Type 1 ECG, shown in Figure 1, is the diagnosed Brugada pattern that is measured in patients with or without medication. Type 2 and type 3 are patterns from potential patients and require further testing.

This study reviews the two most highly debated hypotheses regarding the cause of Brugada syndrome provides a detailed explanation of the mechanisms and published evidence for both the repolarization and depolarization hypotheses. Also, based on the analysis of the evidence provided, we will propose the most likely mechanism behind the cause of the Brugada syndrome.

The following two sections explain a basic heart function and a method of heart diagnosis. The section "Action Potential" has information mainly from the following source: The Action Potential in Ventricular Cells - Phases. (2018, March 8). TeachMePhysiology. The section "Electrocardiograms" is mainly from the following source: CV Physiology | Electrocardiogram (EKG, ECG). (n.d.). Cardiovascular Physiology Concepts. 


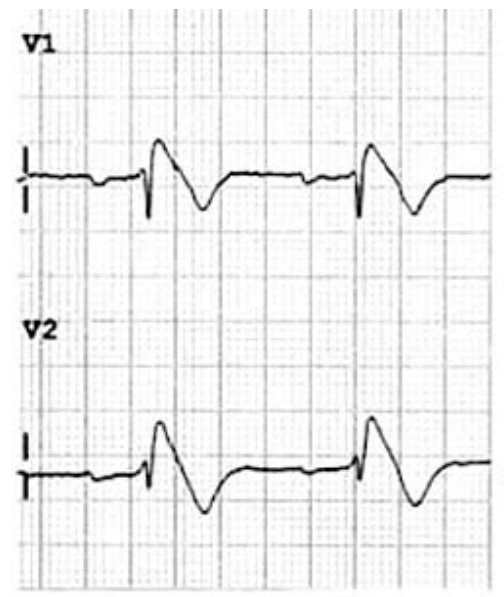

TYPE 1 ECG

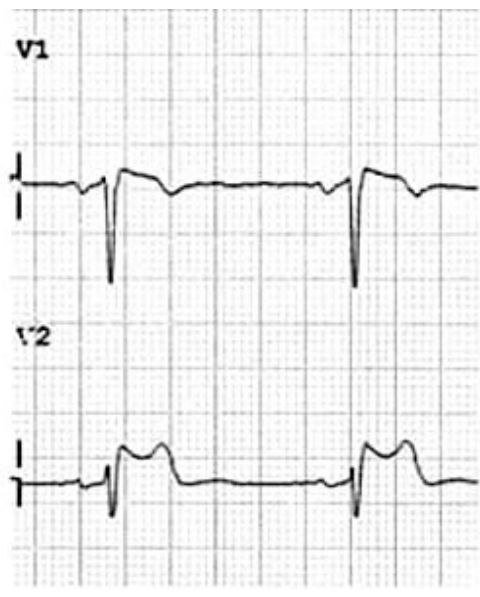

TYPE 2 ECG

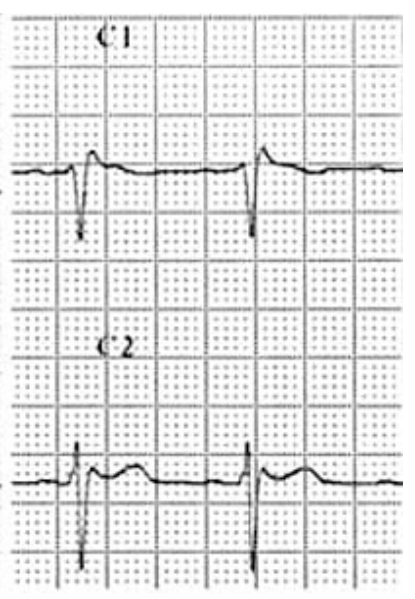

TYPE 3 ECG

Figure 1. 3 Types of Brugada ECG. Source: Larkin, J. (2020). Brugada Syndrome. Life in the Fastlane.

\section{Action Potential}

Action potential (AP) is a process that helps neurons transport chemical signals from one neuron to another. After each stimulus, voltage is carried across the cellular membrane; however, in order to be stimulated, a threshold stimulus for AP must be reached. Neurons are long cells capable of carrying the voltage from one end of the cell to the other. AP travels from the dendrites of a neuron (location of the neuron that receives information) to the axon terminals (ends of the neuron). Gap junctions are sites that relay information from one neuron to another. Connexins are a family of proteins that allow diffusional movement of substances between cells and are an important part of neurons. There are 5 phases (phases 0-4) in the process of AP as illustrated in Figure 2.

\section{Phase 4: Resting Phase}

The resting phase has a transmembrane potential (TMP) of $-90 \mathrm{mV}$. In this phase the sodium ion $\left(\mathrm{Na}^{+}\right)$and calcium ion $\left(\mathrm{Ca}^{2+}\right)$ channels are closed.

\section{Phase 0: Depolarization}

The stimulus triggers the cardiomyocyte (heart muscle cell). Several $\mathrm{Na}^{+}$channels, some coded by the SCN5A gene, will open, increasing the TMP until the TMP reaches $-70 \mathrm{mV}$, the threshold potential for cardiomyocytes. After passing the minimum voltage for the AP to run through the muscle cell, a large number of $\mathrm{Na}^{+}$channels will open up, and at $-40 \mathrm{mV}$ L-type $\mathrm{Ca}^{2+}$ channels (LTCC) will also open, rapidly depolarizing TMP to $0 \mathrm{mV}$ and beyond as positive ions enter the cardiomyocyte. The $\mathrm{Na}^{+}$channels are time dependent and will rapidly close afterward.

\section{Phase 1: Early Repolarization}

The overshot TMP is positive and $\mathrm{K}^{+}$channels will open and leak out $\mathrm{K}^{+}$, which will lower the TMP to $0 \mathrm{mV}$.

\section{Phase 2: Plateau}

The inward flow of $\mathrm{Ca}^{2+}$ from the LTCC and outward flow of $\mathrm{K}^{+}$will cause the voltage to plateau.

\section{Phase 3: Repolarization}


The LTCC are deactivated and $\mathrm{K}^{+}$outflow will overcome the $\mathrm{Ca}^{2+}$ inflow, which will bring the TMP back to $-90 \mathrm{mV}$.

Ion channel pumps restore the polarized state of the $\mathrm{Ca}^{2+}, \mathrm{Na}^{+}$, and $\mathrm{K}^{+}$gradients of the cardiomyocyte.

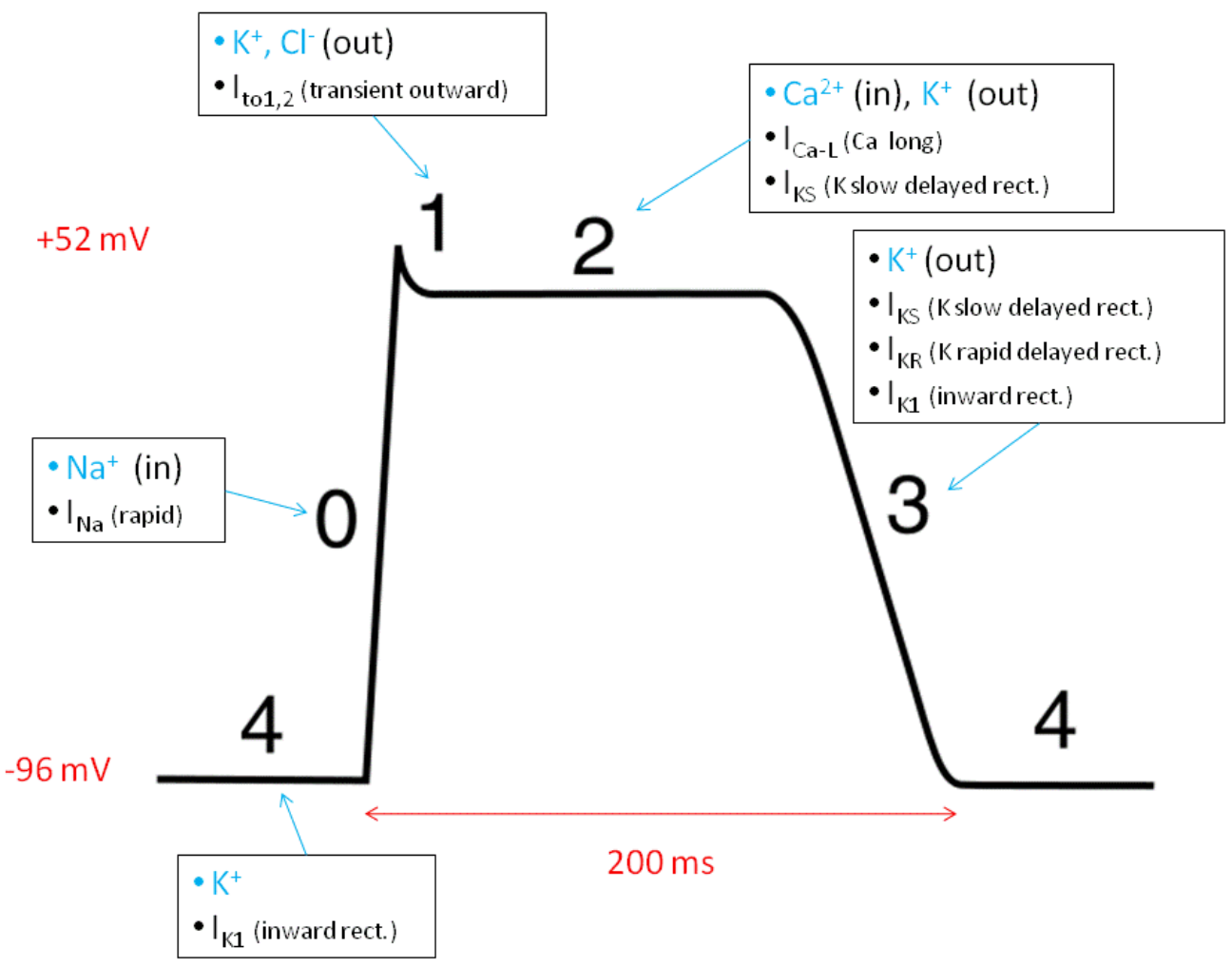

Figure 2. Illustration of cardiac action potential. Source: The Action Potential in Ventricular Cells - Phases. (2018). TeachMePhysiology.

\section{Electrocardiograms}

Electrocardiograms (ECGs or EKGs) are diagrams that show the electrical current generated by the AP activities in the myocardiocytes. ECGs are noninvasive procedures where the electrical activity is measured by a series of electrodes placed on the body surface. The rhythm of the heart is commonly determined by a pacemaker site called the sinoatrial (SA) node located in the posterior wall of the right atrium near the superior vena cava. 


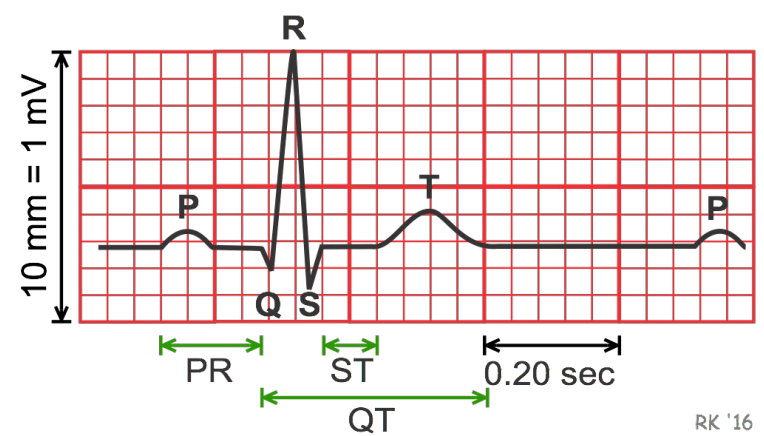

Figure 3. Electrocardiographic segment broken into its respective segments. Source: CV Physiology | Electrocardiogram (EKG, ECG). (n.d.). Cardiovascular Physiology Concepts.

As shown in Figure 3, an electrocardiographic segment is the entire line and is made up of electrocardiographic intervals. Each interval is made up of one or more waves:

The $\mathbf{P}$ wave correlates with the atrial depolarization at the posterior wall of the right atrium near the superior vena cava. Its duration is 0.8 to 0.10 seconds.

The PR interval is the period of time from the beginning of the $\mathrm{P}$ wave to beginning of the QRS complex. The interval lines up with the start of atrial and ventricular depolarization. It represents the atrial depolarization and the physical delay of the atrioventricular (AV) node. It usually lasts 0.12 to 0.20 seconds.

The QRS complex represents the total time of ventricular depolarization. The shape of the QRS complex varies depending on the electrodes used when taking the ECG and/or during abnormal conduction. Normally it ranges from 0.06 to 0.10 seconds.

The T wave represents ventricular repolarization and is occasionally followed by a U wave that arises from the remaining ventricular repolarization.

The ST segment is after the QRS complex and lasts until the beginning of the T wave. Here, both ventricles are depolarized. The ST segment correlates with phase 2 of AP.

The QT interval covers the period where both ventricular depolarization and repolarization take place. It ranges from 0.20 to 0.40 seconds.

The various parts of the ECG wave correspond with the electric activities in the heart. ECGs are studied to detect any abnormalities in a heart's electrical system. Abnormal tests relating to the syndrome are categorized into the three types of Brugada ECG, mentioned in Figure 1, to determine whether a patient needs treatment or further testing.

\section{Repolarization Hypothesis}

The repolarization hypothesis mainly addresses the AP that makes up the electrical conduction that carries the electric current which pumps the heart (Meregalli at al., 2005). Brugada syndrome is an abnormality in the heart's steady beating that is believed to be the result of the action potential duration (APD, the period of time the AP's voltage is above a certain voltage) varying between the inside layer (the endocardium) and outside of the heart's muscular tissue (epicardium) (Tse et al., 2016).

Repolarization and its relation to the type 1 ECG has been shown in canine wedge preparations, or heart samples from canines. If the transmural dispersion of repolarization, or repolarization across an organic wall or blood 
vessel, shows up on the type 1 ECG, there is an extreme chance for arrhythmia or there is an extreme arrhythmogenicity (Tse et al., 2016). Although the type 1 ECG pattern is associated with arrhythmias not all patients with Brugada syndrome will experience arrhythmias (Wilde et al., 2010; Antzelevitch, 2006; Yan and Antzelevitch, 1999).

During the diagnosis process, patients with the type 2 or type 3 ECG patterns take further tests to include the diagnostic type 1 ECG with the aid of drugs (Tse et al., 2016). Patients undergoing such tests are unlikely to experience sudden or extreme arrhythmogenicity of transmural dispersion or repolarization that is found in the canine wedge preparations (Wilde et al., 2010). Such repolarization situations would require either the complete splitting of the endocardium and epicardium from the myocardium or the slight slowing down of conduction of electricity and shift in APD in the heart caused by structural abnormalities. The latter is more logical proving the mechanism of repolarization (Morita et al., 2008; Wilde et al., 2010).

The ECG pattern of Brugada shows a slowing down of conduction in the right ventricle (RV), which results in the late activation of the right ventricle outflow tract (RVOT), where blood passes to leave the right ventricle into the arteries (Wilde et al., 2010). The RVOT AP is in comparison delayed to the rest of the RV AP. The disorganized activation leads to arrhythmia (Wilde et al., 2010). Evidence from ECGs support the repolarization hypothesis because of the elevated ST segment. (Zhang et al. 2015). During the hatched phase in Figure 4D, there is more positive potential, and less work needs to be done in the RV than the RVOT. It causes an intercellular current to flow to the RVOT, neutralizing the imbalance in membrane potential (Wilde et al., 2010; Meregalli et al., 2005). However, the abnormal electrical pulse runs in a circuit, through the extracellular space, and the current rebounds back to the RV from the RVOT. The closed-circuit results in a positive signal (recorded positive voltage) at an electrode positioned over the RVOT and an elevated ST-segment (Wilde et al., 2010).

T-wave alternans, or QRS complex amplitude, which is the axis between heart beats or the wandering baseline, also supports the hypothesis (Nishizaki et al., 2005; Uchimura-Makita et al., 2014; Sakamoto et al., 2015). The T-wave is negative in Figure 4F and during the hatched phase, the membrane potential gradients of the RV and RVOT are reversed (Wilde et al., 2010; Meregalli et al., 2005). The RVOT membrane potential is more positive than the RV's. This starts the small circuit with the current traveling from RVOT to the RV through the right precordial lead, resulting in the expected negative T-wave (Wilde et al., 2010).

The slow conduction delay in the RVOT as the RV and RVOT exchange currents will accelerate the RVOT AP (Franz et al., 1987). The delay in conduction most likely contributes to the Brugada syndrome phenotype, but also involves the mild structural defects present in some Brugada cases, especially the loss of function of sodium current $\left(\mathrm{I}_{\mathrm{Na}}\right.$ ) involved in arrhythmia cases (Wilde et al., 2010).

Mutations to the SCN5A gene or any gene related to $\mathrm{I}_{\mathrm{Na}}$ and the RV AP or RVOT AP can cause loss-offunction to the associated proteins, affecting the AP speed by either speeding up or slowing down activation of ion channels in the AP circuit (Veldkamp et al., 2000). This causes the delay in the conduction by the differences in APD of the epicardium and endocardium. This difference occurs when the APD of the epicardium is shortened with a higher cardiac transient outward K current ( $\mathrm{I}_{\mathrm{to}}$ ) (Tse et al., 2016). The $\mathrm{I}_{\mathrm{to}}$ and $\mathrm{I}_{\mathrm{Na}}$ are interrelated in the AP process.

During phase 1 of AP, the imbalance of ionic current is due to the late/failed activation of LTCC. This shows up in the ECG and the loss of the AP dome. Phase 2 is hypothesized to be involved with the transferring of the electric signal between the epicardium and the endocardium, also known as electrotonic coupling (Tse et al., 2016; Yan and Antzelevitch, 1999). This is also the cause of abnormal heartbeat in normal individuals, extrasystole, because of the excitation of the myocytes, or muscular tissue, involved in the beating of the heart (Yan and Antzelevitch, 1999). The phase 2 reentry happens in the sub-epicardial layer. This is from the quick repolarization reducing the phase 2 period of the AP (Maoz et al., 2014). Reentry will disrupt the heart's electrical circuit's flow and result in arrhythmia (Lukas and Antzelevitch, 1996). On the ECG it will show as a repolarization gradient reversal. The ST segment will show up elevated and the T-wave will be inverted. 
A

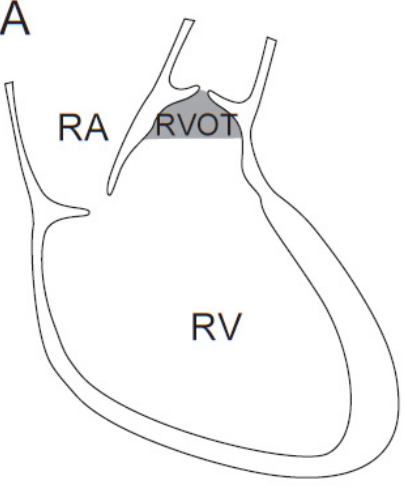

C

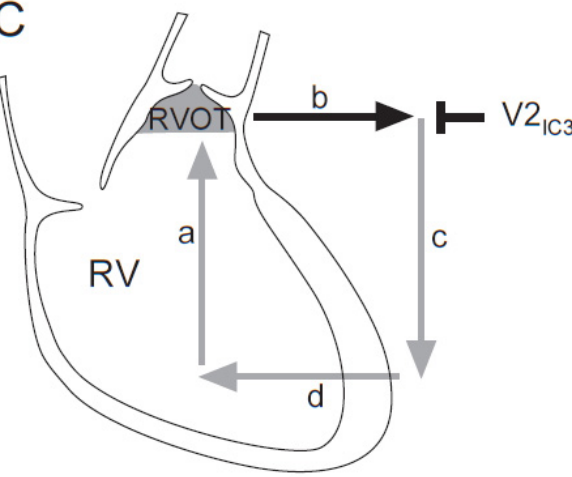

E

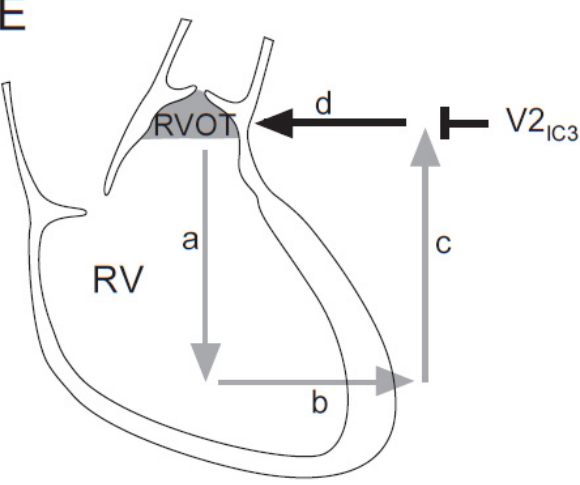

B

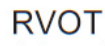

delayed

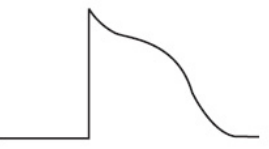

RV

early

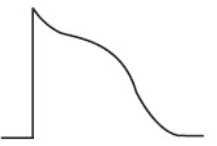

D

RV

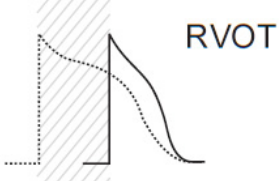

F

RV
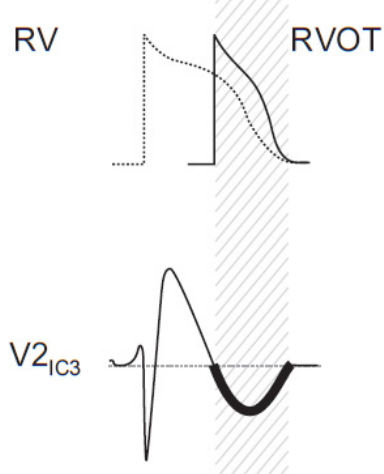

Figure 4. Models for type 1 Brugada syndrome. Source: Meregalli et al. (2005). Pathophysiological Mechanisms of Brugada Syndrome: Depolarization Disorder, Repolarization Disorder, or More?. Cardiovascular Research.

\section{Depolarization Hypothesis}

The depolarization hypothesis also addresses the AP that makes up the heart's electrical circuit. However, while the repolarization hypothesis involves the shortening of the APD, the depolarization hypothesis involves the reduced conduction velocity (CV) of AP phase 0 (Tse et al., 2016).

Evidence towards depolarization is mainly from clinical and modeling studies (Meregalli at al., 2005). Mutations to the SCN5A channel in experimental mice showed reduced CV (Leoni et al., 2010; Boukens et al., 2013; Schweizer et al., 2013). CV is associated with interstitial fibrosis, or scarred tissue. $\mathrm{Na}^{+}$channels "co-localise with 
gap junctions or of intercalated disks," which are structural formations of myocardial cells, resulting in a vast interacting network of $\mathrm{Na}^{+}$channels, gap junctions (Cohen, 1996; Tse et al., 2016). Gap junction components are dependent on each other. This is evident in the increased myocardial fibrosis from collagen deposition, or wound healing, and reduced gap junction expressions in the right ventricle outflow tract (RVOT) (Tse et al., 2016).

Fundamentally, there is a conduction delay in the RVOT in comparison to the RV AP (Figure 4B; Meregalli et al, 2005). Epicardial electrograms measuring the conus branch of the coronary artery found running over the RVOT surface show an activation delay (Nagase et al., 2002). The membrane potential, in turn, is more positive than the RVOT, driving the current to RVOT and neutralizing the membrane potential between the two uneven layers of cells (Meregalli at al., 2005). When the signal is picked up by the lead V2IC3, the electrode over the RVOT in an electrocardiogram, there is a positive signal that elevates the ST segment on the ECG. The current then flows back to the RV to complete the circuit. This wavebreak results in a negative T-wave as the current flows away from V2IC3 (Meregalli et al., 2005).

When the large differences in membrane potential exist in the ischemic, areas of decreased blood flow, and non-ischemic zones, ventricular tachyarrhythmia (VT) from Brugada syndrome stem from between the early and delayed depolarizations (Janse et al., 1981). The ventricular late potentials that delay and break up the ventricular conduction in the RV and RVOT are strong indicators of future ventricular arrhythmias and are linked with VF and VT episodes, which are common in Brugada syndrome (Ikeda et al., 2001; Takami et al., 2003). The late potentials are also connected to the ST segment elevation related to the syndrome.

Another study showed that a catheter ablation of the RVOT, a procedure used to modify heart tissue, with Brugada syndrome provided a normal ECG with no evident Brugada pattern and the heart did not experience further VT and VF attacks (Tse et al., 2016; Nademanee et al., 2011; Brugada et al., 2015). The slowing down of conduction in the RV is essential to the type Brugada syndrome in the ECG (Postema et al., 2010). This is why after the catheter ablation, the modified heart tissue ceased to show any more signs of Brugada syndrome (Tse et al., 2016; Meregalli at al., 2005). The reduced connexin, an important part of gap junctions, results from decreased $\mathrm{I}_{\mathrm{Na}}$ responsible for the reduced velocity (Postema et al., 2010). In turn, the reduced conduction and closed circuit may result in VT or VF episodes (Tse et al., 2016).

\section{Conclusion}

There are many pieces of evidence behind each of the proposed mechanisms of the Brugada syndrome as many diseases develop and occur through multiple mechanisms. As discussed above, while there is evidence to support both potential hypotheses, the most compelling evidence comes from studies involving human studies of the heart and is in support of the repolarization hypothesis. Models with ischemia are based on the depolarization models, but ischemia does not contribute directly to the syndrome (Wilde et al., 2010). Models for the depolarization hypothesis also demonstrate the repolarization hypothesis instead. Mutations to the $\mathrm{Na}^{+}, \mathrm{K}^{+}$and $\mathrm{Ca}^{2+}$ channels and arrhythmia occur in a way that is consistent with the repolarization hypothesis. Alterations to the $\mathrm{Na}^{+}$channels in the experiments do not help in supporting the depolarization hypothesis (Wilde et al., 2010).

When the $\mathrm{I}_{\text {to }}$ gradient removed, the Brugada syndrome's mechanisms will be blocked, elevating the STsegment and the disappearance of late potentials and inhibition of $\mathrm{I}_{\mathrm{Na}}$ by quinidine show point to the effects of Quinidine, a medical antiarrhythmic drug that blocks $\mathrm{I}_{\text {to }}$, helps prevent reduced arrhythmias in patients (Meregalli et al., 2005; Wilde et al., 2010). Quinidine can be used to treat the repolarization heterogeneity.

Furthermore, ECGs type 1, indicate that ECG type 1 Brugada syndrome show patterns of repolarization (Zhang et al., 2015). Steep repolarization gradients were present in the study. Although many patients do not experience arrhythmias, they do have a constant type 1 ECG. It establishes the predominance of repolarization over depolarization in not only patients who know they have Brugada syndrome but patients that have yet to experience an arrhythmia attack. 
This study comprehended the different mechanisms behind the Brugada syndrome and evaluated through the evidence of the real-life studies, ECG, and application of medication on $\mathrm{I}_{\mathrm{Na}}$ and $\mathrm{I}_{\mathrm{to}}$. Given the current evidence and the evaluation of the two different hypotheses, repolarization is most likely the main mechanism behind the Brugada syndrome.

\section{Acknowledgments}

Clair Koo would like to thank her dad for being her inspiration to write this paper and her teacher, Ms. Tarn Wilson for her guidance and mentorship.

\section{References}

Antzelevitch, C. (2006). Brugada Syndrome. Pacing and Clinical Electrophysiology, 29(10), 1130-1159. https://doi.org/10.1111/j.1540-8159.2006.00507.x

Boukens, B. J., Sylva, M., de Gier-de Vries, C., Remme, C. A., Bezzina, C. R., Christoffels, V. M., \& Coronel, R. (2013). Reduced Sodium Channel Function Unmasks Residual Embryonic Slow Conduction in the Adult Right Ventricular Outflow Tract. Circulation Research, 113(2), 137-141. https://doi.org/10.1161/circresaha.113.301565

Brugada, J., Pappone, C., Berruezo, A., Vicedomini, G., Manguso, F., Ciconte, G., Giannelli, L., \& Santinelli, V. (2015). Brugada Syndrome Phenotype Elimination by Epicardial Substrate Ablation. Circulation: Arrhythmia and Electrophysiology, 8(6), 1373-1381. https://doi.org/10.1161/circep.115.003220

Brugada Syndrome. (2016, April 4). NORD (National Organization for Rare Disorders). https://rarediseases.org/rare-diseases/brugada-syndrome/

Brugada syndrome - I. Symptoms and causes. (2020). Mayo Clinic. https://www.mayoclinic.org/diseases-conditions/brugada-syndrome/symptoms-causes/syc-20370489

Brugada syndrome - II. Diagnosis and treatment - Mayo Clinic. (2020). Mayo Clinic. https://www.mayoclinic.org/diseases-conditions/brugada-syndrome/diagnosis-treatment/drc-20370494

Cohen, S. A. (1996). Immunocytochemical Localization of rH1 Sodium Channel in Adult Rat Heart Atria and Ventricle. Circulation, 94(12), 3083-3086. https://doi.org/10.1161/01.cir.94.12.3083

CV Physiology | Electrocardiogram (EKG, ECG). (n.d.). Cardiovascular Physiology Concepts. https://www.cvphysiology.com/Arrhythmias/A009

Franz, M. R., Bargheer, K., Rafflenbeul, W., Haverich, A., \& Lichtlen, P. R. (1987). Monophasic Action Potential Mapping in Human Subjects with Normal Electrocardiograms: Direct Evidence for the Genesis of the T Wave. Circulation, 75(2), 379-386. https://doi.org/10.1161/01.cir.75.2.379

Ikeda, T., Sakurada, H., Sakabe, K., Sakata, T., Takami, M., Tezuka, N., Nakae, T., Noro, M., Enjoji, Y., Tejima, T., Sugi, K., \& Yamaguchi, T. (2001). Assessment of Noninvasive Markers in Identifying Patients at Risk in The Brugada Syndrome: Insight into Risk Stratification. Journal of the American College of Cardiology, 37(6), 1628-1634. https://doi.org/10.1016/s0735-1097(01)01197-4 
Janse, M. J. \& Kléber, A. G. (1981). Electrophysiological Changes and Ventricular Arrhythmias in the Early Phase of Regional Myocardial Ischemia. Circulation Research, 49(5), 1069-1081. https://doi.org/10.1161/01.res.49.5.1069

Larkin, J. (2020, September 23). Brugada Syndrome. Life in the Fastlane. https://litfl.com/brugada-syndrome-ecg$\underline{\text { library/ }}$

Le Scouarnec, S., Karakachoff, M., Gourraud, J.-B., Lindenbaum, P., Bonnaud, S., Portero, V., Duboscq-Bidot, L., Daumy, X., Simonet, F., Teusan, R., Baron, E., Violleau, J., Persyn, E., Bellanger, L., Barc, J., Chatel, S., Martins, R., Mabo, P., Sacher, F., Haïssaguerre, M., Kyndt, F., Schmitt, S., Bézieau, S., Le Marec, H., Dina, C., Schott, J., Probst, V., \& Redon, R. (2015). Testing the Burden of Rare Variation in Arrhythmia-Susceptibility Genes Provides New Insights into Molecular Diagnosis for Brugada Syndrome. Human Molecular Genetics, 24(10), $2757-2763$. https://doi.org/10.1093/hmg/ddv036

Leoni, A.-L., Gavillet, B., Rougier, J.-S., Marionneau, C., Probst, V., Le Scouarnec, S., Schott, J.-J., Demolombe, S., Bruneval, P., Huang, C. L. H., Colledge, W. H., Grace, A. A., Le Marec, H., Wilde, A. A., Mohler, P. J., Escande, D., Abriel, H., \& Charpentier, F. (2010). Variable Nav1.5 Protein Expression from the Wild-Type Allele Correlates with the Penetrance of Cardiac Conduction Disease in the Scn5a+/- Mouse Model. PLoS ONE, 5(2), e9298. https://doi.org/10.1371/journal.pone.0009298

Lukas, A. \& Antzelevitch, C. (1996). Phase 2 Reentry as a Mechanism of Initiation of Circus Movement Reentry in Canine Epicardium Exposed to Simulated Ischemia. Cardiovascular Research, 32(3), 593-603.

https://doi.org/10.1016/s0008-6363(96)00115-0

Maoz, A., Christini, D. J., \& Krogh-Madsen, T. (2014). Dependence of Phase-2 Reentry and Repolarization Dispersion on Epicardial and Transmural Ionic Heterogeneity: a Simulation Study. EP Europace, 16(3), 458-465.

https://doi.org/10.1093/europace/eut379

Meregalli, P., Wilde, A., \& Tan, H. (2005). Pathophysiological Mechanisms of Brugada Syndrome: Depolarization Disorder, Repolarization Disorder, or More?. Cardiovascular Research, 67(3), 367-378.

https://doi.org/10.1016/j.cardiores.2005.03.005

Morita, H., Zipes, D. P., Fukushima-Kusano, K., Nagase, S., Nakamura, K., Morita, S. T., Ohe, T., \& Wu, J. (2008). Repolarization Heterogeneity in the Right Ventricular Outflow Tact: Correlation with Ventricular Arrhythmias in Brugada Patients and in an in Vitro Canine Brugada Model. Heart Rhythm, 5(5), 725-733. https://doi.org/10.1016/j.hrthm.2008.02.028

Nademanee, K., Veerakul, G., Chandanamattha, P., Chaothawee, L., Ariyachaipanich, A., Jirasirirojanakorn, K., Likittanasombat, K., Bhuripanyo, K., \& Ngarmukos, T. (2011). Prevention of Ventricular Fibrillation Episodes in Brugada Syndrome by Catheter Ablation Over the Anterior Right Ventricular Outflow Tract Epicardium. Circulation, 123(12), 1270-1279. https://doi.org/10.1161/circulationaha.110.972612

Nagase, S., Kusano, K. F., Morita, H., Fujimoto, Y., Kakishita, M., Nakamura, K., Emori, T., Matsubara, H., \& Ohe, T. (2002). Epicardial Electrogram of the Right Ventricular Outflow Tract in Patients with the Brugada Syndrome. Journal of the American College of Cardiology, 39(12), 1992-1995. https://doi.org/10.1016/s0735-1097(02)01888-0

Nishizaki, M., Fujii, H., Sakurada, H., Kimura, A., \& Hiraoka, M. (2005). Spontaneous T Wave Alternans in a Patient with Brugada Syndrome-Responses to Intravenous Administration of Class I Antiarrhythmic Drug, Glucose 
Tolerance Test, and Atrial Pacing. Journal of Cardiovascular Electrophysiology, 16(2), 217-220. https://doi.org/10.1046/j.1540-8167.2004.40411.x

Postema, P. G., van Dessel, P. F. H. M., Kors, J. A., Linnenbank, A. C., van Herpen, G., Ritsema van Eck, H. J., van Geloven, N., de Bakker, J. M. T., Wilde, A. A. M., \& Tan, H. L. (2010). Local Depolarization Abnormalities Are the Dominant Pathophysiologic Mechanism for Type 1 Electrocardiogram in Brugada Syndrome. Journal of the American College of Cardiology, 55(8), 789-797. https://doi.org/10.1016/j.jacc.2009.11.033

Sakamoto, S., Takagi, M., Tatsumi, H., Doi, A., Sugioka, K., Hanatani, A., \& Yoshiyama, M. (2015). Utility of TWave Alternans During Night Time as a Predictor for Ventricular Fibrillation in Patients with Brugada Syndrome. Heart and Vessels, 31(6), 947-956. https://doi.org/10.1007/s00380-015-0692-y

Schweizer, P. A., Fink, T., Yampolsky, P., Seyler, C., Fabritz, L., Kirchhof, P., Becker, R., Koenen, M., Katus, H. A., \& Thomas, D. (2013). Generation and Characterization of SCN5A Loss-of-Function Mutant Mice Modeling Human Brugada Syndrome. European Heart Journal, 34(suppl 1), 4556. https://doi.org/10.1093/eurheartj/eht310.4556

Takami, M., Ikeda, T., Enjoji, Y., \& Sugi, K. (2003). Relationship Between ST-Segment Morphology and Conduction Disturbances Detected by Signal-Averaged Electrocardiography in Brugada Syndrome. Annals of Noninvasive Electrocardiology, 8(1), 30-36. https://doi.org/10.1046/j.1542-474x.2003.08106.x

The Action Potential in Ventricular Cells - Phases. (2018, March 8). TeachMePhysiology. https://teachmephysiology.com/cardiovascular-system/cardiac-muscle/action-potential-ventricular-cells/

Tse, G., Liu, T., Li, K. H. C., Laxton, V., Chan, Y. W. F., Keung, W., Li, R. A., \& Yan, B. P. (2016). Electrophysiological Mechanisms of Brugada Syndrome: Insights from Pre-clinical and Clinical Studies. Frontiers in Physiology, 7, 1-6. https://doi.org/10.3389/fphys.2016.00467

Uchimura-Makita, Y., Nakano, Y., Tokuyama, T., Fujiwara, M., Watanabe, Y., Sairaku, A., Kawazoe, H., Matsumura, H., Oda, N., Ikanaga, H., Motoda, C., Kajihara, K., Oda, N., Verrier, R. L., \& Kihara, Y. (2014). Time-Domain T-Wave Alternans is Strongly Associated with a History of Ventricular Fibrillation in Patients with Brugada Syndrome. Journal of Cardiovascular Electrophysiology, 25(9), 1021-1027. https://doi.org/10.1111/jce.12441

Veldkamp, M. W., Viswanathan, P. C., Bezzina, C., Baartscheer, A., Wilde, A. A. M., \& Balser, J. R. (2000). Two Distinct Congenital Arrhythmias Evoked by a Multidysfunctional Na ${ }^{+}$Channel. Circulation Research, 86(9), E91E97. https://doi.org/10.1161/01.res.86.9.e91

Wilde, A. A. M., Postema, P. G., Di Diego, J. M., Viskin, S., Morita, H., Fish, J. M., \& Antzelevitch, C. (2010). The Pathophysiological Mechanism Underlying Brugada Syndrome. Journal of Molecular and Cellular Cardiology, 49(4), 543-553. https://doi.org/10.1016/j.yjmcc.2010.07.012

Yan, G.-X. \& Antzelevitch, C. (1999). Cellular Basis for the Brugada Syndrome and Other Mechanisms of Arrhythmogenesis Associated With ST-Segment Elevation. Circulation, 100(15), 1660-1666.

https://doi.org/10.1161/01.cir.100.15.1660

Zhang, J., Sacher, F., Hoffmayer, K., O’Hara, T., Strom, M., Cuculich, P., Silva, J., Cooper, D., Faddis, M., Hocini, M., Haïssaguerre, M., Scheinman, M., \& Rudy, Y. (2015). Cardiac Electrophysiological Substrate Underlying the 
ECG Phenotype and Electrogram Abnormalities in Brugada Syndrome Patients. Circulation, 131(22), 1950-1959. https://doi.org/10.1161/circulationaha.114.013698 